\title{
Characteristics of lateral vehicular interactions in heterogeneous traffic with weak lane discipline
}

\author{
Anuj Kishor Budhkar ${ }^{1}$ Akhilesh Kumar Maurya ${ }^{1}$
}

Received: 19 September 2016/Revised: 14 March 2017/Accepted: 18 April 2017/Published online: 10 May 2017

(C) The Author(s) 2017. This article is an open access publication

\begin{abstract}
Heterogeneous traffic conditions prevail in developing countries. Vehicles maintain weak lane discipline which increases lateral interactions of vehicles significantly. It is necessary to study these interactions in the form of maintained lateral gaps for modeling this traffic scenario. This paper aims at determining lateral clearances maintained by different vehicle types while moving in a heterogeneous traffic stream during overtaking. These data were collected using an instrumented vehicle which runs as a part of the stream. Variation of obtained clearance with average speed of interacting vehicles is studied and modeled. Different instrumented vehicles of various types are developed using (1) ultrasonic sensors fixed on both sides of vehicle, which provide inter-vehicular lateral distance and relative speed; and (2) GPS device with cameras, which provides vehicle type and speed of interacting vehicles. They are driven on different roads in six cities of India, to measure lateral gaps maintained with different interacting vehicles at different speeds. Relationships between lateral gaps and speed are modeled as regression lines with positive slopes and beta-distributed residuals. Nature of these graphs (i.e., slopes, intercepts, residuals) are also evaluated and compared for different interacting vehicle-type pairs. It is observed that similar vehicle pairs maintain less lateral clearance than dissimilar vehicle pairs. If a vehicle interacts with two vehicles (one on each side) simultaneously, lateral clearance is reduced and safety of
\end{abstract}

Anuj Kishor Budhkar anuj.budhkar@iitg.ernet.in

Akhilesh Kumar Maurya maurya@iitg.ernet.in

1 Department of Civil Engineering, Indian Institute of Technology Guwahati, Guwahati, Assam 781039, India the vehicles is compromised. The obtained relationships can be used for simulating lateral clearance maintaining behavior of vehicles in heterogeneous traffic.

Keywords Ultrasonic sensors - Heterogeneous traffic · Lateral gaps - Lateral vehicular interactions - Weak lane discipline

\section{Introduction}

Traffic conditions in developing countries are characterized by two prominent phenomena-(1) heterogeneous vehicle types and (2) weak lane discipline. Due to these conditions, a vehicle's behavior in such a traffic stream is impacted by the actions of not only the leading vehicles but also adjacent vehicles. Thus, maneuvering a vehicle needs more attentive control to avoid accidents and involves greater interactions between vehicles present in close neighborhood of the vehicle-laterally (sidewise) as well as longitudinally. Till recently, majority of works have been carried out to study the longitudinal interaction (or carfollowing behavior) of vehicles in different traffic streams; however, less research has been devoted to lateral interactions between vehicles. Therefore, a detailed research on lateral interactions between vehicles needs to be carried out for better understanding of traffic conditions in developing countries.

Lateral clearance (LC) is the sidewise safety spacing maintained by a vehicle with neighboring vehicles when it travels through a traffic stream. Along with the longitudinal gap, LC is an important parameter in heterogeneous traffic streams with weak lane discipline, and is affected by several factors including type of vehicle, vehicle speed, driver behavior and external factors. Heterogeneous traffic results 
in larger variation in individual vehicle's speed and no lane-discipline behavior during overtaking or interacting with other vehicles. Thus, a study is needed which develops a model of LC for each type of vehicles, or rather, between pairs of different vehicle types. Previous studies by Gunay [1] made consideration for loose lane discipline and function of available width for overtaking vehicles, whereas Pal and Mallikarjuna [2] and Mallikarjuna et al. [3] presented a rough estimation of lateral interaction between vehicles using image processing-based vehicle detection software. However, these studies lack in largescale accurate data collection from aforesaid traffic streams.

This paper attempts to model the relationship of LC between a pair of vehicles moving in the same direction, with their speeds. The speed of the test/instrumented vehicle is calculated using a high-precision global positioning system (GPS)-based data logger, whereas lateral distances and relative speed are calculated using ultrasonic sensors. The scope of this study is limited to uninterrupted mid-block sections which are not affected by external features.

The rest of this paper is organized as follows. Section 2 presents a literature review on previous studies regarding lateral interactions. Section 3 elaborates methodology of data collection and extraction. Section 4 states field sites used for data collection. Section 5 describes the modeling of obtained data, vehicle-type-wise behavior, variation of road width, interaction with multiple vehicles and use of obtained model. The last section summarizes the main findings and future scope of this study.

\section{Literature review}

The work on lateral vehicular interactions can be traced from May's experiments [4], which calculated internal frictions between two vehicles on test track. Based upon traffic arrival pattern of a multi-lane unidirectional highway, Mahalel and Hakkert [5] concluded that arrival pattern of vehicles in one lane is dependent on the arrival patterns of vehicles in the other lanes. For mixed traffic condition, there is no restriction of lateral movement, and hence, vehicles have the freedom to traverse in any gaps without the need to travel in demarcated paths. Hence, lateral movement for overtaking is not only in the form of lane changing (which has large literature), but also smaller lateral shifts which would be sufficient enough to maintain a clearance between adjacent vehicles as per the comfort of drivers. In this regard, clearance gaps between vehicles on bidirectional roads were first evaluated by Nagaraj et al. [6] elaborates using video-recording method, but the less accurate technology used then motivates the need for larger and versatile data samples.

\subsection{Previous work on staggered car-following}

Staggered car-following behavior is predominant in developing countries, where limited attempts have been made to study the relationship between lateral and longitudinal distance between interacting vehicles. Gunay [7] defined the term lane-based driving discipline as the tendency to drive within a lane by keeping to the center as closely as possible (unless in lane changing). Gunay [1] remarked that when two vehicles travel parallel to each other, they tend to shy away. A new car-following relationship which is based on staggered car-following is developed, where the lateral frictional discomfort between moving vehicles is taken into account. Maximum escape speed (MES) or speed with which vehicle can safely overtake other vehicle, depending upon available road width, was calculated. It was found that there is a seconddegree relationship between vehicle speed and road width available for overtaking. Further, a simulation model was developed [8] based upon observations drawn from field data collection. Gunay and Erdemir [9] analyzed staggered time headways between neighboring vehicles and found that drivers prefer to pass or lag behind the vehicle in the adjacent lane, rather than driving side by side.

\subsection{Previous work on speed-LC relationship}

Some research has been carried out to establish the relationship between speed and lateral (or transverse) clearance maintained by interacting vehicles. Pal and Mallikarjuna [2] collected data and evaluated the average lateral gap versus percentage area occupancy relationship in heterogeneous traffic. In a later study [3], it was found that lateral gaps maintained by vehicles vary with respect to their speeds and vehicle types. A commercial software 'TRAZER' which automatically collects traffic data based upon image processing of a recorded traffic stream was used in this research; however, data extracted from this software suffers from serious accuracy issues at different levels. A continuum model by Nair et al. [10] or a pore space model by Ambarwati et al. [11] has been devised based upon available minimum spacing between vehicle corners, referred as pores. These models do not consider vehicle speeds in developing relationships of distances between vehicles with other traffic parameters like area occupancy. Linear relationship between LC and speed was assumed in many heterogeneous simulation models, such as the HETEROSIM model developed by Arasan and Koshy [12], the CASIM model by Maurya and Chakraborty [13] and the unidirectional model by Metkari et al. [14]. Validation of these models was limited to macroscopic parameters only. Potential field model (conceived by Chakraborty et al. [15]) can also be extended for heterogeneous 
conditions, if different vehicle-type parameters are introduced and lateral parameters are calibrated properly.

Previous car-following models have not considered any lateral terms in the equations. Recent research work by Delpiano et al. [16] involves introduction of a term called collateral anomaly. A study has been made to see the effect of neighboring leading vehicle's position on the following vehicle in a staggered car-following. Attempts have also been made to incorporate the staggered car-following behavior using the visual angle concept by Jin et al. $[17,18]$, but these approaches do not adequately represent the lateral discomfort. Recent study by Pal and Mallikarjuna [19] used effective width of vehicles (which also included LC maintained by vehicles) to calculate macroscopic parameter of area occupancy of traffic. Munigety et al. [20] have not ventured into modeling speeds with respect to LC with the change in road and vehicle parameters. More recent work by Dimayacyac and Palmiano [21] focuses on average relationship between LC and speed for various vehicle pairs, but the deviation of $\mathrm{LC}$ at similar speeds is not studied. The popular commercial vehicle simulator VISSIM [22] allows for input of average LC values for particular vehicle type at stopped condition $(0 \mathrm{~km} / \mathrm{h})$ and at $50 \mathrm{~km} / \mathrm{h}$ speeds. However, there is no highlight on vehicle pairwise variation, or deviation at similar speed levels.

\subsection{Use of instrumented vehicles for traffic data extraction}

There is a need to study the average LC maintained between vehicles, during the entire overtaking process. Data collection on LC maintained by the interacting vehicles in such traffic streams is quite challenging. Static traffic-recording techniques (for example, video recording) can provide LC between vehicles, only at a particular section. The average clearance during the entire overtaking may not be captured. In order to capture this, there is a need to develop an instrumented vehicle which will be a part of the traffic stream and measure clearances of other vehicles with itself using sensing devices. Optical sensors were used by Wong and Qidwai [23] for collision avoidance of a car using vehicle electronic control unit. Based on this approach, an instrumented vehicle was developed using ultrasonic sensors for data collection in this work. Such an instrumented vehicle was also developed by Venter and Knoetze [24] for measuring LCs between bikes and other vehicles in order to predict the safe width of bike lanes. Various sensors can also be used for vehicle detection systems. Literature for video image or vision-based sensors has been reviewed by Sun et al. [25]. Moving vehicle detection and classification system is popular, with video image or vision-based sensors being used on a large scale.

It can be concluded from the literature review presented in above subsections that majority of studies are restricted to the study of lane-changing behavior in traffic stream with lane discipline. Limited attempts have been made to study the LC aspects in heterogeneous traffic stream with weak lane discipline. These studies have certain issues such as limitation of observed data points, accuracy issues, or results being difficult to reproduce and replicate because of the cumbersome data extraction process. This motivates the authors to collect largescale LC data for verity of interacting vehicles' pairs of heterogeneous traffic streams with weak lane discipline using sophisticated equipments. Further attempts also have been made to model the relationship between speed of interacting vehicles and LC maintained between them.

\section{Methodology for field data collection and analysis}

The methodology for field data collection that includes the concept of development of an instrumented vehicle consists of sensors and V-box assembly, extracting different parameters from collected data, and file-handling for getting data in the final form. In order to measure speed and LC simultaneously, a synchronized setup consisting of a sensor assembly (to measure distances) and a GPS device (to measure speeds) with video cameras was used (refer to Fig. 1). The following subsections describe calculation of test and interacting vehicles' speed, LC between them and vehicle-type determination.

\subsection{Speed of test vehicle}

Test vehicle speed is calculated using video V-box manufactured by 'Racelogic.' This is an accelerometer with a GPS data logger and two traffic-recording cameras. It updates vehicle position from satellite signals and calculates speed at every $10 \mathrm{~Hz}$ frequency.

\subsection{LC between test and interacting vehicles}

LC between vehicles, which is denoted by $C$ in the context, is calculated using a set of six ultrasonic sensors fitted on both sides of test vehicles as shown in Fig. 1. The ultrasonic sensors are operated and controlled by a microcontroller board. The microcontroller and sensor setup is shown in Fig. 1a. The program code is written in an open project Arduino 1.0.5.

A sensor triggers an ultrasonic pulse at the speed of sound, which is reflected back after hitting any obstacle. 

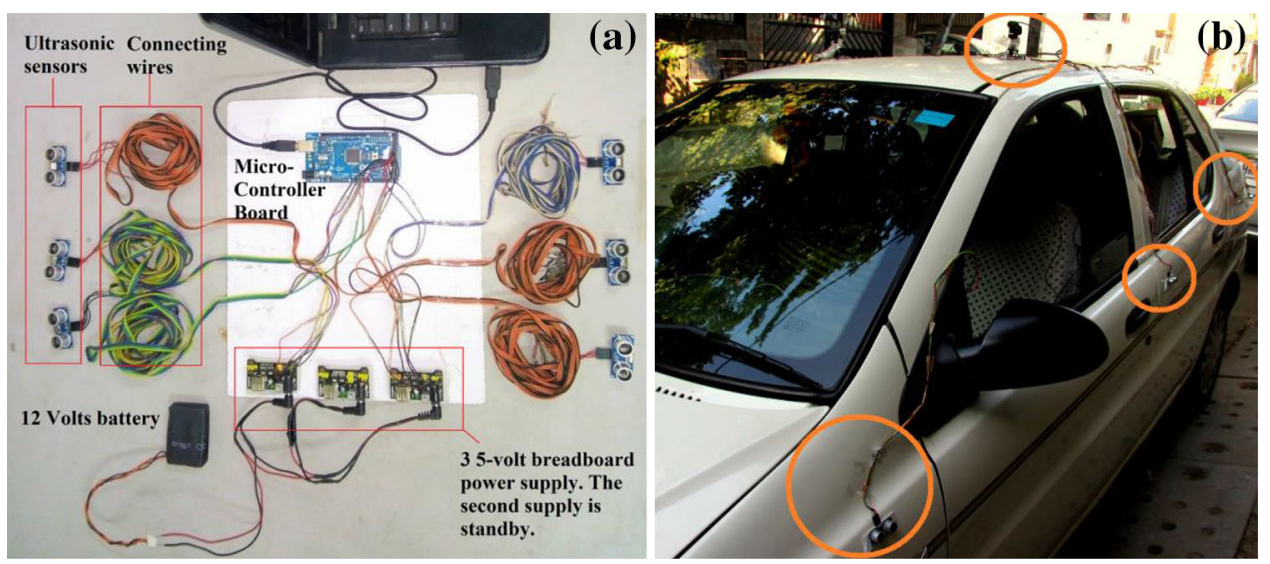

Fig. 1 Microcontroller and sensor setup used for data collection (a) and installed sensors on vehicle (b)

When a pulse is triggered, detectors in the sensors search the reflected echo, whereas the microcontroller board keeps a track of time lag. Totally six sensors are fixed on vehicles during data-collection period (three on each side of the test vehicle) as shown in Fig. 1b. The distance between neighboring vehicle and sensor is calculated by time-offlight, that is, half of the product of the speed of sound and the time required by waves from emitter to detector. The distance between two neighboring sensors is measured as inter-sensor distance. Each sensor emits pulses in a conical direction. Based on the pre-calibrated cone-angle and the distance between interacting vehicles, a correction to intersensor distance is made dynamically. For example, in Fig. 2, the corrected inter-sensor distance between sensors 1 and 2 is $\mathrm{AL}$ if the other vehicle is overtaking the test vehicle, and $\mathrm{CN}$ if the test vehicle is overtaking the other vehicle. The line AR in Fig. 2a represents the path followed by the edge of the interacting vehicle. If echo pulse is not detected, or if the object is beyond the stipulated

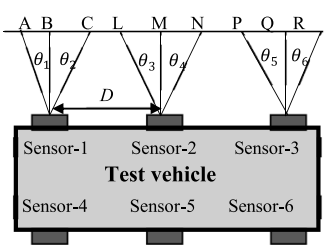

Direction of travel (a)

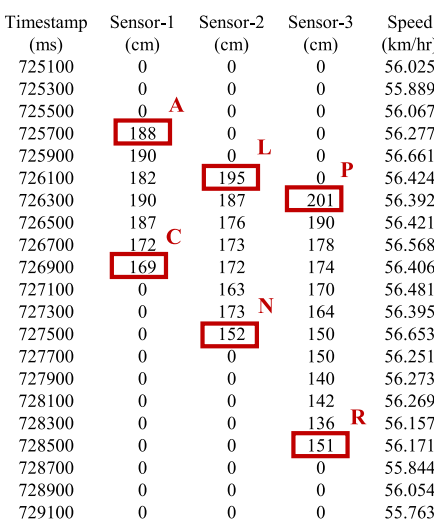

(b)
Fig. 2 Sensor positioning (a) and sample reading obtained in sensor and V-box data-collection file (b) range of sensors, then the sensors are programmed to return a 'null' reading. Ultrasonic pulses are set to trigger and receive pulses at $5 \mathrm{~Hz}$ frequency intervals.

\subsection{Speed of interacting vehicle}

The relative speed of the interacting vehicle is calculated from Eq. (1) based on the test vehicle speed, inter-sensor distance and sensor time stamps of vehicle detection. Thus, the speed of interacting vehicle $\left(v_{\mathrm{I}}\right)$ can be calculated, once the speed of test vehicle $\left(v_{\mathrm{T}}\right)$ during this interaction is known.

$v_{\mathrm{I}}=v_{\mathrm{T}} \pm \frac{D}{\Delta t}$,

where $D$ denotes the corrected inter-sensor distance and $\Delta t$ denotes the difference in sensor time stamps of vehicle detection.

If the interacting vehicle is overtaking the test vehicle, the relative speed is added to the test vehicle's speed; else, it is subtracted from the test vehicle's speed. V-box and sensor setup run independently and collect data at similar frequencies. Hence, initial readings (starting datum) of both the equipments are synchronized by matching with a global time.

\subsection{Determination of type of interacting vehicles}

It is difficult to distinguish whether the echoed ultrasonic pulses received by the detectors are reflected from other neighboring vehicles or any other obstacles (like median, street furniture, etc.) solely on the basis of obtained sensor readings. To segregate actual interaction readings, observer needs to manually identify vehicle types with their approximate times of interactions. For this purpose, the video recorded by the two attached cameras focused on either sides helps in identifying vehicle type of interacting 
vehicle and its approximate time of interaction for each side. This time of interaction is already synchronized in GPS data logger. While identifying, vehicles were classified into various categories like cars, buses, trucks, light commercial vehicles (LCV), motorized three-wheelers (auto) and motorized two-wheelers (bike). The urban traffic in the study stretch consisted of majority of these vehicle types, and the clubbing into these categories is on the basis of similarity in vehicle characteristics (such as size, engine properties, steering capability).

\subsection{File-handling and processing of collected data}

Initially, a file (say, File 'A') is generated by microcontroller. It contains readings of six sensors along with time stamp. Another file is generated by V-box (File ' $\mathrm{B}$ ') which contains speed of vehicle with time stamp. These two files (File A and File B) are merged in a single file (File ' $\mathrm{X}$ ') by synchronizing with the global time stamp present in each file. A sample of readings in File ' $\mathrm{X}$ ' is shown in Fig. 2b. It basically contains instantaneous speeds at every $5 \mathrm{~Hz}$ and corresponding obtained distances for all three sensors at those instances. A separate file (File ' $\mathrm{Y}$ ') contains the manually identified vehicle types of interacting vehicles, time stamps of their interactions and indicators- $-(+1$ or -1 ) for overtaking the test vehicle or getting overtaken by the test vehicle, respectively. There is a minor variation $(<10 \%)$ in the LC obtained between instantaneous sensor readings over one particular interaction. The authors are interested in studying the average maintained value of LC during the entire overtaking period and not instantaneous values. Hence, data from Files ' $\mathrm{X}$ ' and ' $\mathrm{Y}$ ' are merged by averaging sensor readings from all the three sensors (of one side of the test vehicle), each averaged over time stamps corresponding to the particular interaction. Similarly, speed of the test vehicle for that interaction is calculated by averaging all instantaneous speeds during one interaction. The entire process of file-handling is shown in Fig. 3. Thus, the final output master-file contains information mainly about vehicle types of test and interacting vehicles, average LC and speeds of both interacting vehicles.

For calculating relative speed, the test vehicle should completely overtake or get overtaken by the interacting vehicle. Previous studies show that vehicles usually do not prefer to move side by side, but generally shy away from each other to stay in a staggered manner while traveling in the neighboring lanes. So in this study, LCs are measured only during overtaking or shying away process of these interacting vehicle pairs. Data obtained with this process are analyzed, and results are presented in the next two sections of this paper.

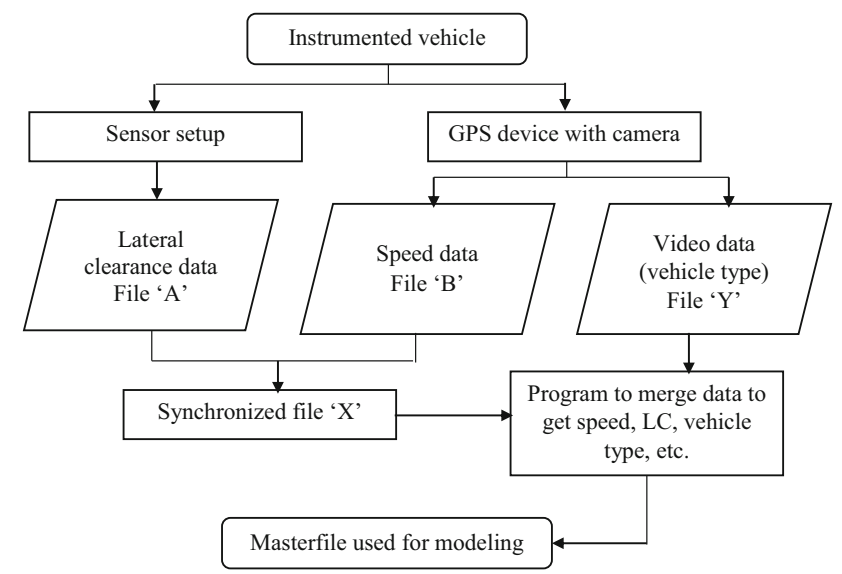

Fig. 3 Schematic diagram of file-processing for data extraction about interacting vehicles

\section{Field data collection}

Test vehicle travels on predefined routes comprising roads with different widths within the city of Delhi, Guwahati, Kolkata, Bengaluru, Pune and Mumbai in India. Data corresponding to mid-block sections (in uninterrupted traffic condition) of roads were segregated for this analysis based on the video recorded by V-box cameras. The road stretches covered under different routes of the cities are mentioned in Table 1.

Data from sensors and V-box readings corresponding to mid-block sections of predefined routes of different cities are separated for the further analysis under this work. Master files for all cities are generated based on the procedure suggested in Fig. 3. During data collection, it was observed that vehicles rarely interact beyond LC of $2.5 \mathrm{~m}$. Therefore, vehicle pairs with a LC more than $2.5 \mathrm{~m}$ were not considered for further study. It is hypothesized that interacting vehicles equally contribute to the maintaining of LC. This will give rise to two fundamental claims: (1) if $x$ and $y$ are two different vehicle types, $C_{(x-y)}$ (LC between vehicles $x$ and $y$ ) is the same as $C_{(y-x)}$; and (2) the average speed of interacting vehicles over the interaction time affects the LC, rather than individual speeds. A total of 6016 vehicle pair interactions were extracted and considered for analysis. Results obtained from data analysis are presented in subsequent sections.

\section{Analysis and results}

Results of the data analysis like LC versus speed relationship, LC variation with interacting vehicle pairs, cities or road width and multiple vehicle interaction are presented in this section. 
Table 1 Details of road stretches and test vehicles used in field data collection in different cities

\begin{tabular}{|c|c|c|c|c|c|c|c|c|c|}
\hline \multirow{2}{*}{$\begin{array}{l}\text { Serial } \\
\text { no. }\end{array}$} & \multirow[t]{2}{*}{ City } & \multirow[t]{2}{*}{ Road stretches } & \multicolumn{7}{|c|}{ Test vehicles driven } \\
\hline & & & $\begin{array}{l}\text { Hatch- } \\
\text { back } \\
\text { car }\end{array}$ & $\begin{array}{l}\text { Sedan } \\
\text { car }\end{array}$ & $\begin{array}{l}\text { SUV } \\
\text { car }\end{array}$ & Van & Auto & Bike & Bus \\
\hline 1 & Delhi & $\begin{array}{l}\text { Outer ring road, Inner ring road, Nelson Mandela road, Africa Ave, } \\
\text { GT road, Mehrauli-Badarpur road, Mehrauli-Gurgaon road, } \\
\text { Charan Singh Road, and DND flyway }\end{array}$ & $\boldsymbol{V}$ & $\boldsymbol{v}$ & $\boldsymbol{V}$ & $\checkmark$ & $\checkmark$ & & \\
\hline 2 & Guwahati & Guwahati bypass, and GS road & $\checkmark$ & $\boldsymbol{v}$ & $\checkmark$ & $\boldsymbol{v}$ & $\boldsymbol{V}$ & $\checkmark$ & $\boldsymbol{V}$ \\
\hline 3 & Kolkata & $\begin{array}{l}\text { EM bypass, VIP road, CIT road, Gariahat road, DPS road } \\
\text { (Tollygunge), Manicktala Vivekananda Road, Chittaranjan Ave, } \\
\text { and Rash Behari Ave }\end{array}$ & $\checkmark$ & $\checkmark$ & $\boldsymbol{v}$ & & $\checkmark$ & $\checkmark$ & \\
\hline 4 & Bengaluru & $\begin{array}{l}\text { Mysore Road, Bellary Road, CV Raman road, West chord road, } \\
\text { Outer Ring Road, Hosur Road, NICE road, TCM Royan road } \\
\text { (Majestic), and Racecourse road }\end{array}$ & $\checkmark$ & $\checkmark$ & $\checkmark$ & & $\boldsymbol{v}$ & $\boldsymbol{V}$ & \\
\hline 5 & Pune & $\begin{array}{l}\text { Bibwewadi Road, Sinhagad Road, Ambedkar-Wellesley road, Old } \\
\text { Bombay-Poona Highway, Pune bypass, Ganeshkhind road, Tilak } \\
\text { road, and Karwe road }\end{array}$ & $\checkmark$ & $\checkmark$ & $\checkmark$ & & $\checkmark$ & $\boldsymbol{v}$ & \\
\hline 6 & Mumbai & $\begin{array}{l}\text { Link road, Western Express Highway, Eastern express highway, } \\
\text { Eastern freeway, Pedder Road, Marine Drive, Bandra Worli Sea } \\
\text { Link, and Jogeshwari-Vikhroli link road }\end{array}$ & $\checkmark$ & $\checkmark$ & $\checkmark$ & & $\checkmark$ & $\checkmark$ & \\
\hline
\end{tabular}

\subsection{Relationship between lateral gap and average speed of interacting vehicle pairs}

The interacting vehicle pairs which have been considered in this study are cars with other cars, autos, two-wheelers, LCVs and heavy vehicles (combined data of buses and trucks); autos with other autos, cars, bikes and heavy vehicles; and bikes with other bikes, autos and cars. Figure 4 presents the LC $C$ (in $\mathrm{cm}$ ) versus average speed $\bar{v}$ (in $\mathrm{km} / \mathrm{h}$ ) data points for all combination of interacting vehicles mentioned above. It is clear that LC increases with the average speed of interacting vehicles. Further, different interacting vehicle pairs maintain a range of LC at a particular average speed. This $C-\bar{v}$ relationship can be represented by a statistical linear model. A linear model is chosen since increasing the degree of equation does not improve the fitting of regression curve.

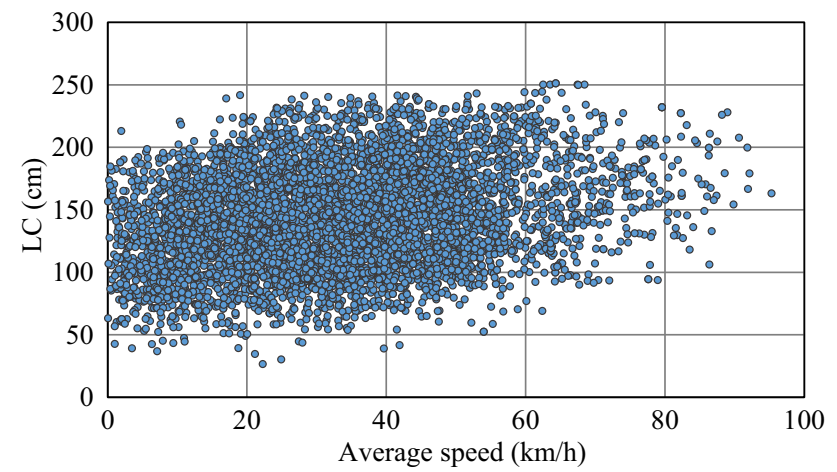

Fig. 4 Scatter plot of average speed $(\bar{v})$ versus LC $(C)$ between all interacting pairs of vehicles, combined data for all cities in this study
Therefore, the model proposed for $C-\bar{v}$ relationship comprises a deterministic part (regression line) and a stochastic part (residual distribution). General equation of this model is represented as Eq. (2),

$C=S \bar{v}+I+\varphi$,

where $\varphi$ is residual terms about the mean, $S$ is slope, and $I$ is intercept. Several common distributions (normal, lognormal, beta, log-logistic, etc.) are fitted against the residual distribution, and it is observed that the distribution of residual terms about the regression line is observed to follow a distribution statistically similar $(p=0.063)$ to beta distribution. The comparison of various distributions with the residuals from field data along with their $p$ values is provided in Fig. 5. General form of beta distribution is given in Eq. (3):

$f(x)=\frac{1}{B\left(\alpha_{1}, \alpha_{2}\right)} \frac{(x-a)^{\alpha_{1}-1}(b-x)^{\alpha_{2}-1}}{(b-a)^{\alpha_{1}+\alpha_{2}-1}}$,

where $B\left(\alpha_{1}, \alpha_{2}\right)=\int_{0}^{1} t^{\alpha_{1}-1}(1-t)^{\alpha_{2}-1} \mathrm{~d} t\left(\alpha_{1}, \alpha_{2}>0\right)$.

Here, $f(x)$ represents the frequency distribution function of beta distribution. The general form of beta distribution is determined by four parameters namely $a, b, \alpha_{1}$, and $\alpha_{2}$. The coefficients $a$ and $b$ determine the range of residual spread, and $\alpha_{1}$ and $\alpha_{2}$ are the shape parameters. $B\left(\alpha_{1}, \alpha_{2}\right)$ is the beta function expressed as a function of shape parameters. Beta distribution is a close-ended distribution with maximum and minimum thresholds on maintenance of LC. Upper and lower values of these thresholds can be calculated by adding values of coefficients $a$ and $b$ of beta distribution, respectively, to the obtained LC by regression line. The 


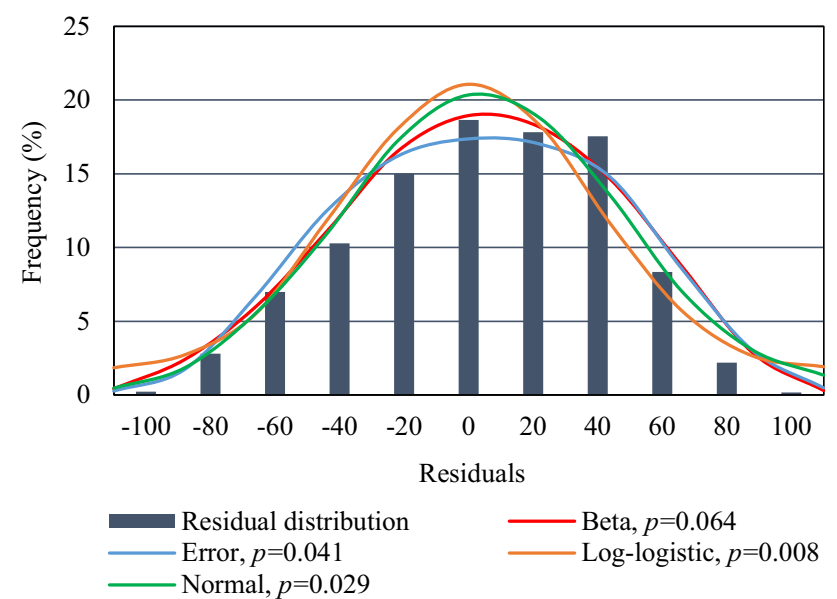

Fig. 5 Comparison of various common distributions with residual plot of obtained field data

phenomenon is also observed in real traffic, since vehicles would not venture below or above a certain threshold for safe maneuvering. Since driver behavior is a natural phenomenon, distribution of residuals is close to normal distribution $(p=0.03$ for all combined data of all vehicle types). Residuals also need to be checked for heteroscedasticity and autocorrelation. Results of GoldfeldQuandt (GQ) test for heteroscedasticity ( $F$ test of $F_{\mathrm{GQ}}$ ) derive $F_{\mathrm{GQ}}$ for lower 3/8th data and middle 1/4th data as 1.113 , whereas $F_{\mathrm{GQ}}$ for middle $1 / 4$ th and higher $3 / 8$ th data as 1.003 . These values are less than the critical $F$ value of $3 / 8$ th and $1 / 4$ th data (i.e., 1503 and 2256 , respectively), denoted as $F_{\mathrm{c}}$, at significance level of $0.01\left(F_{\mathrm{c}}=1.115\right)$.

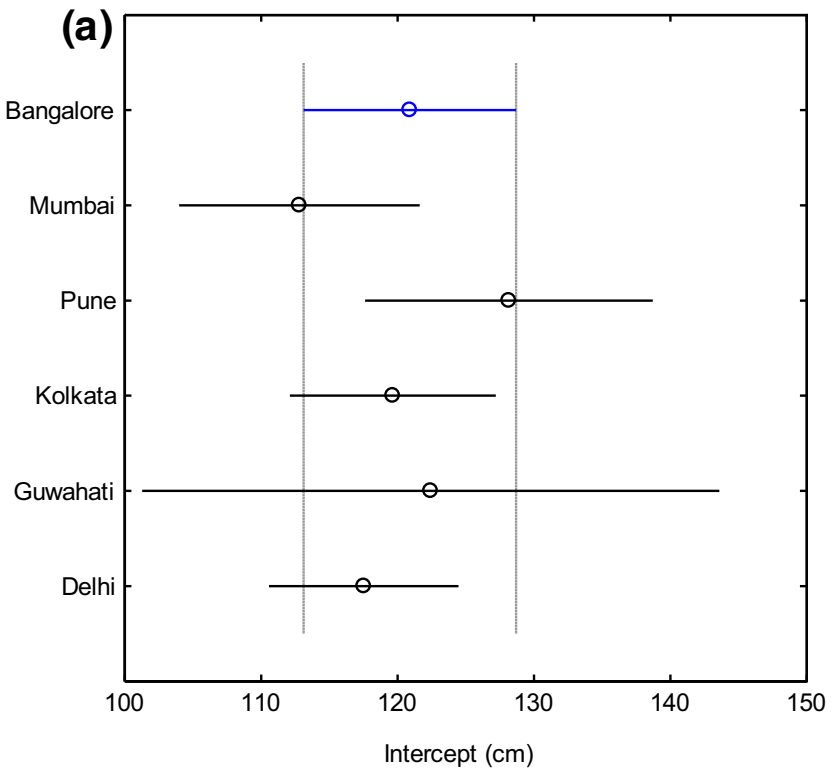

The value of Durbin Watson statistic (autocorrelation test) is 1.86. Since the value is close to 2 , it means very less autocorrelation between consecutive residual terms. Thus, residuals are found to be homoscedastic with very less autocorrelation. It indicates that the spread of data about the regression line remains consistent with speed. This is because the available road width for overtaking does not change, so drivers traveling at lower speeds also have a wide range of LCs to choose from.

In the current study, $C$ versus $\bar{v}$ data are collected from six different cities and presented in Fig. 4. In order to validate whether the $C$ versus $\bar{v}$ varies significantly with locations (different cities), site-specific data are evaluated and results are presented in the next subsection.

\subsection{Evaluation of city-wise $C$ versus $\bar{v}$ data}

It was observed from Fig. 4 that the LC maintained by interacting vehicles from different cities show increasing trends with average speed of interacting vehicles. The combined deterministic linear relationship between $C$ and $\bar{v}$ for all interacting vehicle pairs observed in all locations together can be represented as LC $C=0.615 \bar{v}+120.883$. However, this observed relationship may change from one city to other due to change in drivers and vehicles' characteristics. A multiple comparison test, i.e., analysis of covariance (ANCOVA) is conducted to evaluate the behavioral change in $C-\bar{v}$ relationship among the interacting vehicle pairs of each city and results are presented in Fig. 6 .

Figure 6 provides the spread of obtained slopes and intercepts of best-fit regression lines for each city. It is

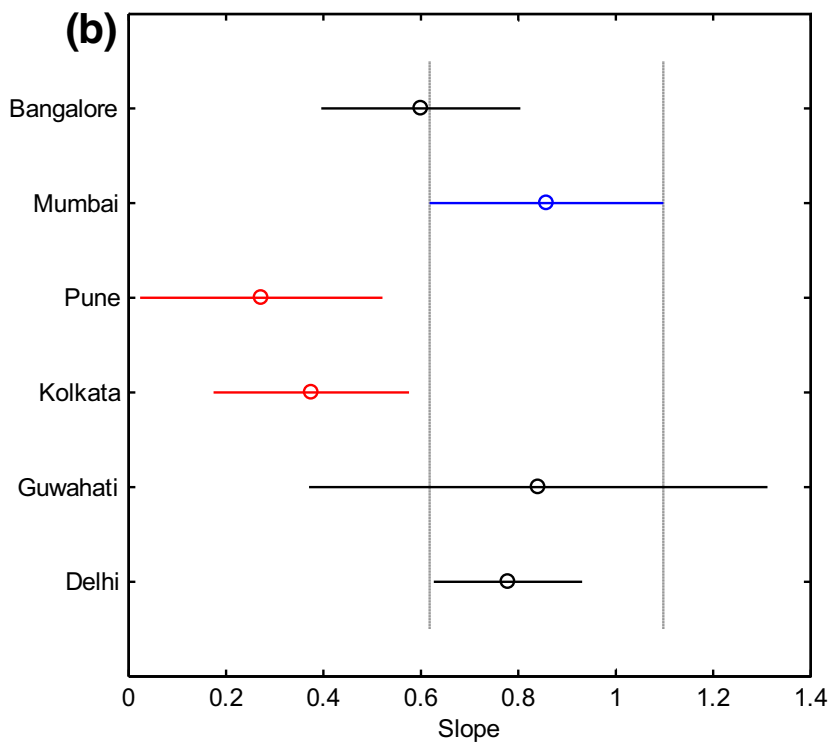

Fig. 6 Comparison of range of estimated slopes and intercepts of LC versus average speed relationships between interacting vehicle pairs moving in different cities. a Test for variation of intercepts for different cities. b Test for variation of slopes for different cities 
Table 2 Statistical comparison of $C$ versus $\bar{v}$ relationship for vehicles from different cities

\begin{tabular}{|c|c|c|c|c|c|c|c|c|c|}
\hline \multirow[t]{2}{*}{ City } & \multirow[t]{2}{*}{ Sample size } & \multicolumn{4}{|c|}{ Intercept (All cities' estimate: 120.883, SE: 2.232) } & \multicolumn{4}{|c|}{ Slope (All cities' estimate: 0.615, SE: 0.053 ) } \\
\hline & & Deviation & SE & $T$ & Prob $>|T|$ & Deviation & SE & $T$ & Prob $>|T|$ \\
\hline Bengaluru & 1310 & 0.123 & 3.854 & 0.032 & 0.975 & -0.025 & 0.099 & -0.250 & 0.803 \\
\hline Mumbai & 886 & -8.101 & 4.288 & -1.889 & 0.059 & 0.256 & 0.113 & 2.263 & 0.024 \\
\hline Pune & 918 & 9.671 & 4.971 & 1.945 & 0.052 & -0.351 & 0.119 & -2.959 & 0.003 \\
\hline Kolkata & 1174 & -2.565 & 3.757 & -0.683 & 0.495 & -0.188 & 0.097 & -1.933 & 0.053 \\
\hline Guwahati & 565 & 4.223 & 8.069 & 0.523 & 0.601 & 0.143 & 0.183 & 0.781 & 0.435 \\
\hline Delhi & 1163 & -3.351 & 3.51 & -0.955 & 0.340 & 0.164 & 0.077 & 2.134 & 0.033 \\
\hline
\end{tabular}

SE indicates standard error. $T$ indicates $T$-value of the data set under consideration. Values marked in bold indicates rejection of null hypothesis at $5 \%$ significance levels

observed from Fig. 6 that slopes of $C$ - $\bar{v}$ relationship of vehicles in Pune and Kolkata significantly differ with those of Mumbai and Delhi. Guwahati city has higher range of slope and intercept variation, since the predictability of close relationship between $\mathrm{LC}$ and speed is less, a possible reason being lesser data points of Guwahati city.

Table 2 compares $C$ versus $\bar{v}$ relationships for interacting vehicle pairs in different cities, with the relationship developed for combined data of all cities. Figure 7 provides linear plots of fitted regression lines for various cities. Table 2 presents the deviation in slope and intercept values of $C$ versus $\bar{v}$ relationship for each city from those of the estimate of combined data for all cities (the estimate is mentioned in heading of Table 2). It is observed from $p$ values of Table 2 that vehicles maintain statistically similar intercepts of regression lines in all the cities. It means that at lower speeds there is no statistical difference in vehicle behavior in different cities. However, vehicles in Pune and Kolkata maintain significantly lesser slope of LC with average speed as those of other cities, whereas Mumbai and Delhi maintain significantly higher slope; as evident from statistics values marked in bold, in the last column of Table 2. In other words, vehicles of Mumbai and Delhi are statistically more sensitive to speed when maintaining LC, whereas those of Pune and Kolkata are

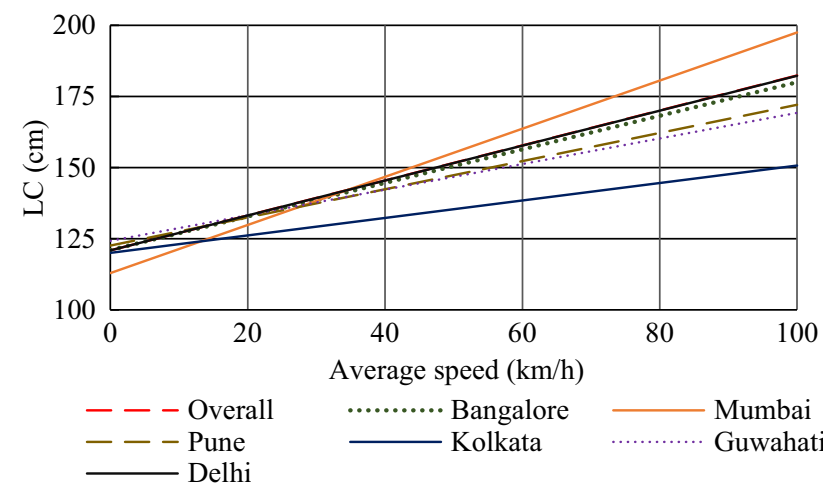

Fig. 7 Comparison of obtained regression lines of LC versus average speed relationship for different cities less sensitive. Since the authors are interested in aggregate behavior of vehicles across all cities, entire dataset of all cities is considered for analysis in subsequent discussions.

\subsection{Vehicle pairwise models for $L C$ versus average speed}

$C$ and $\bar{v}$ are also calculated for certain pairs of vehicles whose data are significantly observed in field. Buses and trucks are considered in a single category as heavy vehicles (HV) since in urban sections studied, noticeable difference in speeds, vehicle sizes and maneuverability were not observed between these two vehicle types. Different interacting vehicle pairs which are included in this study are cars with other cars, bikes, autos, HV and LCVs; autos with other autos, cars, bikes and HVs; and bikes with other bikes. Other vehicle pairs are not considered due to less sample size observed in collected data. Before proceeding for modeling, it is checked whether there is any significant difference among the relationships between different pairs of vehicles keeping one interacting vehicle-type constant. These test results are presented in Table 3. Italicized heading for each vehicle type in Table 3 presents the intercept and slope estimates for overall behavior of that vehicle type with other interacting vehicles. It can be observed that bikes maintain lesser gap (with other vehicles) than cars and autos (with other vehicles). Further, it can be observed that although cars and autos maintains similar gaps at lower speeds; however, autos (with other vehicles) maintain larger gaps at higher speeds.

The rows under each vehicle type represent deviation in intercept and slope estimate for a particular interacting vehicle pair with respect to the overall behavior of that vehicle type. The overall behavior is mentioned in italicized text before pairwise interactions. $T$-value of pairs maintaining significantly different behavior than overall behavior is mentioned in bold. It is observed that autos behave significantly different with cars as compared with other vehicles. Due to high maneuverability of autos, cars maintain higher gaps with them even at lower speeds. 
Table 3 Comparison of $C$ and $\bar{v}$ for different vehicle pairs with their overall behavior

\begin{tabular}{|c|c|c|c|c|c|c|c|c|}
\hline \multirow[t]{2}{*}{ Pair } & \multicolumn{4}{|c|}{ Intercept $(I)$} & \multicolumn{4}{|l|}{ Slope $(S)$} \\
\hline & Deviation & $\mathrm{SE}(\sigma)$ & $T$ & Prob $>|T|$ & Deviation & $\mathrm{SE}(\sigma)$ & $T$ & Prob $>|T|$ \\
\hline \multicolumn{9}{|c|}{ Autos with other vehicles: $I=129.6(\sigma=5.2), S=0.962(\sigma=0.2)$} \\
\hline Auto-Car & 7.555 & 6.67 & 1.133 & 0.258 & -0.516 & 0.242 & -2.128 & $\mathbf{0 . 0 3 4}$ \\
\hline Auto-Bike & -7.504 & 7.327 & -1.024 & 0.306 & 0.205 & 0.272 & 0.754 & 0.451 \\
\hline Auto-Auto & -0.872 & 9.337 & -0.093 & 0.926 & 0.171 & 0.372 & 0.461 & 0.645 \\
\hline Auto-HV & 0.821 & 11.791 & 0.07 & 0.945 & 0.139 & 0.457 & 0.305 & 0.760 \\
\hline \multicolumn{9}{|c|}{ Bikes with other vehicles: $I=119.76(\sigma=6.2), S=0.541(\sigma=0.2)$} \\
\hline Car-Bike & 5.461 & 6.328 & 0.863 & 0.388 & 0.069 & 0.207 & 0.334 & 0.738 \\
\hline Auto-Bike & -3.728 & 6.668 & -0.559 & 0.576 & 0.248 & 0.218 & 1.14 & 0.255 \\
\hline Bike-Bike & -1.733 & 12.019 & -0.144 & 0.885 & -0.317 & 0.396 & -0.8 & 0.424 \\
\hline \multicolumn{9}{|c|}{ Cars with other vehicles: $I=126.51(\sigma=1.9), S=0.550(\sigma=0.05)$} \\
\hline Car-Car & -9.125 & 2.392 & -3.415 & 0.001 & 0.128 & 0.064 & 1.994 & 0.046 \\
\hline Car-Bike & -1.297 & 2.655 & -0.488 & 0.625 & 0.061 & 0.076 & 0.801 & 0.423 \\
\hline Car-Auto & -2.482 & 3.024 & -0.821 & 0.412 & 0.096 & 0.085 & 1.124 & 0.261 \\
\hline Car-HV & -0.657 & 3.511 & -0.187 & 0.851 & 0.144 & 0.094 & 1.521 & 0.128 \\
\hline Car-LCV & 13.561 & 6.209 & 2.184 & 0.029 & -0.429 & 0.173 & -2.478 & 0.013 \\
\hline
\end{tabular}

Data in bold highlights the $T$-value of vehicle pairs maintaining significantly difference behavior than the overall behavior

Similar observation can be made in case of car-bike interactions. These comparison results of regression lines of $C$ and $\bar{v}$ are also mentioned in Fig. 8a-c. Cars are observed to behave significantly different with themselves as compared to other vehicles.

Linear statistical equations are modeled. Model parameters (slope, intercept, coefficients of beta distribution) are calculated for each pair and mentioned in Table 4.

Obtained parameters from Table 4 can be used for modeling gap-maintenance behavior of vehicles in heterogeneous traffic stream. One may also compare estimates of average behavior of different types of vehicle pairs, through regression slopes and intercepts presented in Table 4. By this comparison, for a particular average speed, vehicles maintain lesser LC with vehicles of their own vehicle types. This is evident from slope and intercept equations for car-car, bike-bike and auto-auto. Bikes maintain the least LC among the overall behavior of vehicle types of cars, autos and bikes. Cars and autos maintain larger LC with LCVs. However, autos and cars maintain relatively lesser slope (of LC vs. average speed) with heavy vehicles than their own types, since it was observed that heavy vehicles, in spite of their size, were frequently overtaken by other vehicles even taking significant risk, due to their weak maneuverability and acceleration capability, which resulted in their reduced average speeds.

The coefficients $a$ and $b$ of beta distribution represent the spread of data about the best-fit regression line. Since vehicles can predict the behavior of their own vehicle types more accurately than other types, spread of data (indicated by $(a-b)$ about the best-fit line for car-car, auto-auto and bike-bike pairs is lesser than other combinations. Moreover, for all the pair combinations, $|a|<|b|$, or the extent of residual spread toward higher threshold is greater than that of spread toward lesser threshold (due to safety concerns at lower thresholds). The coefficients $\alpha_{1}$ and $\alpha_{2}$ represent nature of distribution about this spread. If $\alpha_{1}>\alpha_{2}$, then the data are more skewed toward the higher thresholds. Lower absolute values of $\alpha_{1}$ and $\alpha_{2}$ indicate flatter distribution. It is observed that for all the vehicle pairs, $\alpha_{2}>\alpha_{1}$ or in other words, more number of vehicles tends to maintain a LC headway closer to the lower threshold. A flatter distribution is obtained when bikes interact with bikes, cars interact with heavier vehicles (LCVs, buses, trucks) and autos interact with autos or heavy vehicles (as inferred from values of $\alpha_{1}$ and $\alpha_{2}$ ). To calculate the goodness of fit of the residual distribution to beta distribution, the K-S test was applied between the best-fit beta distribution and residual distribution; $p$ value of comparison of these two distributions is presented in the last column of Table 4. It is observed that residuals follow beta distribution at good significance.

\subsection{Shying away behavior during interaction of heterogeneous vehicle pairs}

Authors have attempted to verify a hypothesis that the LC maintained between two interacting vehicles is the result of individual contribution of each vehicle. If this hypothesis may hold true, then for a particular average speed between two interacting vehicle types $x$ and $y, C_{(x-y)}$ should be the 

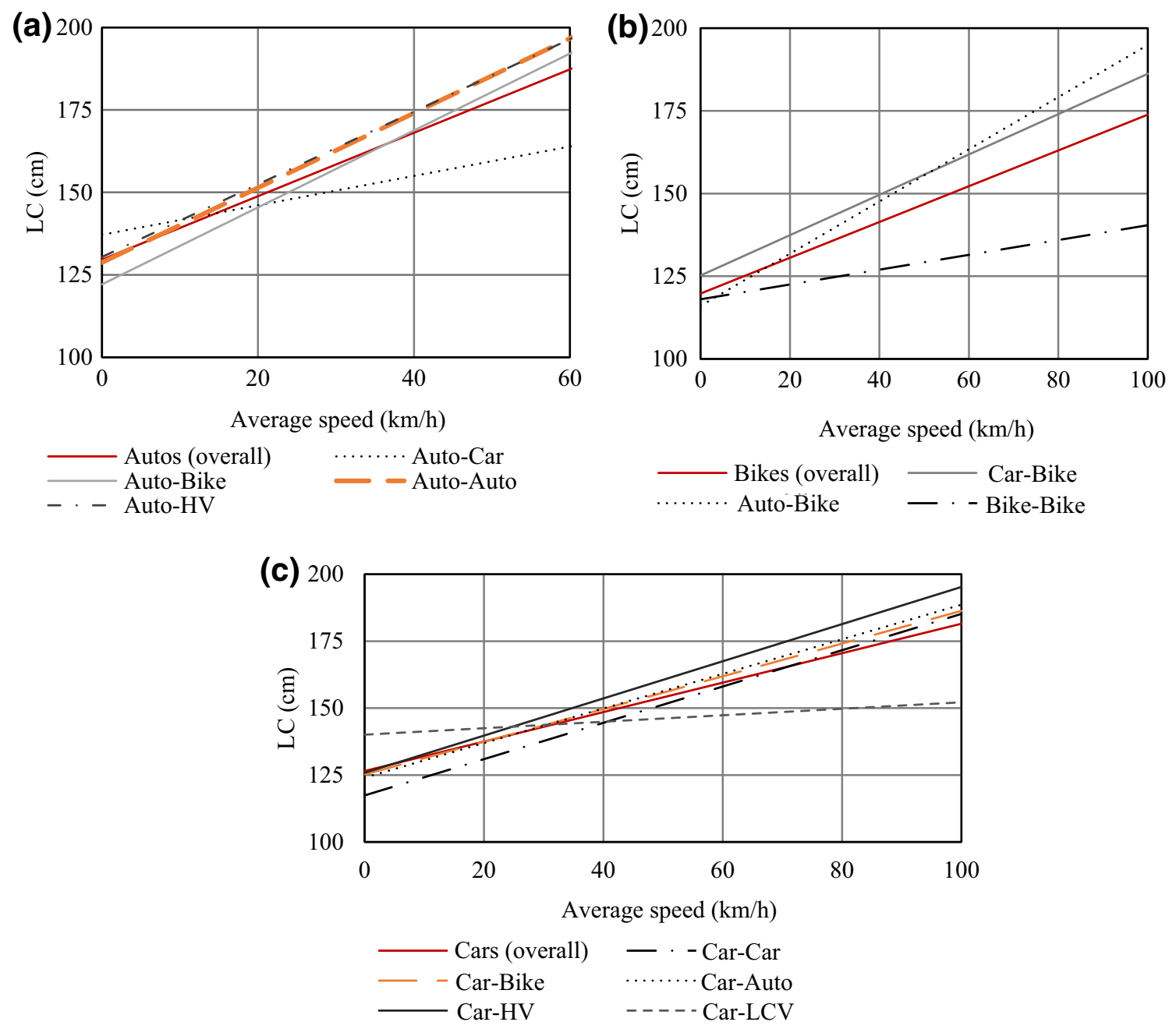

Fig. 8 Comparison of vehicle pair behavior with overall behavior of autos (motorized three-wheelers) (a), bikes (motorized two-wheelers) (b) and cars (c)

Table 4 Model parameter estimation for different vehicle pairs

\begin{tabular}{|c|c|c|c|c|c|c|c|c|}
\hline \multirow[t]{2}{*}{ Vehicle pair } & \multirow[t]{2}{*}{ Sample size } & \multicolumn{2}{|c|}{ Regression line } & \multicolumn{5}{|c|}{ Coefficients of beta distribution } \\
\hline & & Slope & Intercept & $\alpha_{1}$ & $\alpha_{2}$ & $a$ & $b$ & $p$ value of fit \\
\hline All combined & 6016 & 0.641 & 122.3 & 4.496 & 5.671 & -117 & 147.94 & 0.063 \\
\hline Auto-Auto & 140 & 1.037 & 114.55 & 1.861 & 2.338 & -89.79 & 112.73 & 0.787 \\
\hline Auto-Bike & 417 & 0.739 & 117.27 & 3.803 & 4.222 & -120.18 & 133.77 & 0.305 \\
\hline Auto-HV & 131 & 0.985 & 123.86 & 2.235 & 2.633 & -100.97 & 118.31 & 0.892 \\
\hline Bike-Bike & 87 & 0.224 & 118.02 & 1.511 & 1.856 & -68.93 & 84.55 & 0.782 \\
\hline Car-Auto & 897 & 0.642 & 124.12 & 4.263 & 4.995 & -120.59 & 141.41 & 0.600 \\
\hline Car-Bike & 1372 & 0.612 & 125.16 & 6.522 & 8.496 & -131.13 & 170.92 & 0.057 \\
\hline Car-Car & 2246 & 0.681 & 117.31 & 3.971 & 4.891 & -106.90 & 131.94 & 0.116 \\
\hline Car-HV & 538 & 0.675 & 126.35 & 2.264 & 2.789 & -88.39 & 108.85 & 0.997 \\
\hline Car-LCV & 139 & 0.132 & 139.78 & 1.711 & 2.213 & -74.98 & 96.64 & 0.861 \\
\hline
\end{tabular}



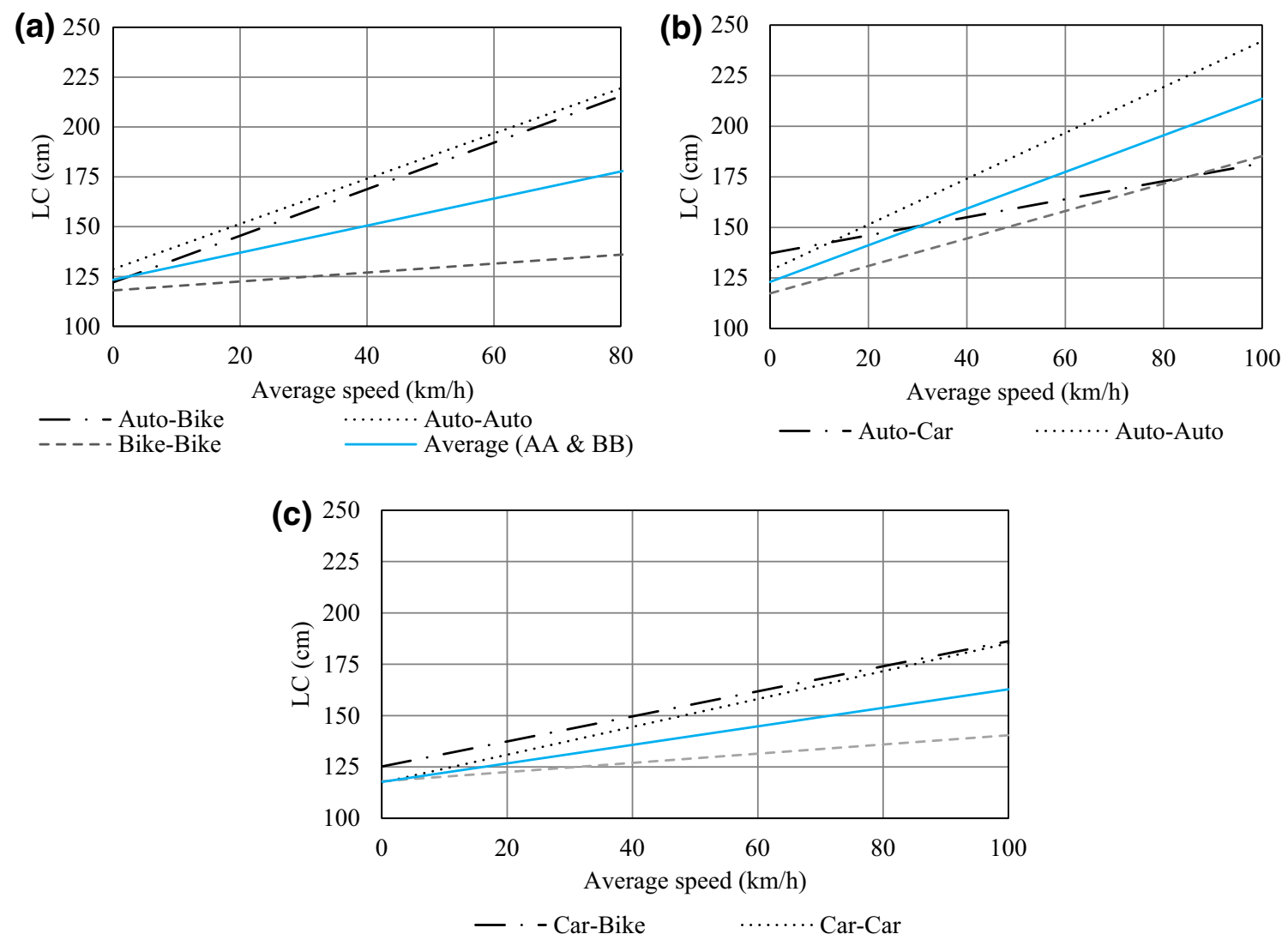

Fig. 9 Comparison of heterogeneous pair interaction with average of respective homogeneous pair interaction for auto-bike (a), auto-car (b) and car-bike interactions (c)

average of $C_{(x-x)}$ and $C_{(y-y)}$. To test this hypothesis, $C-\bar{v}$ relationships (regression lines) observed from field data for homogeneous vehicle pairs (like car-car, bike-bike and auto-auto) are compared with those of heterogeneous vehicle pair combinations (like car-bike, car-auto, autobike). Figure 9 presents the results of this comparison graphically for each vehicle pair. For example, Fig. 9a presents the observed $C-\bar{v}$ relationships for auto-auto (AA), bike-bike $(\mathrm{BB})$ and auto-bike $(\mathrm{AB})$ along with the computed auto-bike $(\mathrm{AB})$ relationship based on the average value of $C_{(\mathrm{AA})}$ and $C_{(\mathrm{BB})}$. It is observed that values of regression lines $C_{(\mathrm{AB})}$ are more than the average value of $C_{(\mathrm{AA})}$ and $C_{(\mathrm{BB})}$. One possible reason for this can be that the driver of one vehicle type is less confident about the behavior of other vehicle types, hence maintains more LC with them than with the same vehicle type. Similar results were obtained when tested for bike and car pairs (refer Fig. 9c). In case of AA and car-car (CC) test, similar result has been observed at lower speeds, but at higher speeds the averaged value of $C_{(\mathrm{AA})}$ and $C_{(\mathrm{CC})}$ is found higher than the field value of $C_{(\mathrm{AC})}$.

From Table 4, for car-auto, bike-auto and car-bike pairs, it can be observed that there is $8.7 \%, 0.85 \%$ and
$6.76 \%$ increase in intercept; and $-25.37 \%, 14.1 \%$ and $35.15 \%$ increase in slope of $C_{(x-y)}$ regression line with speed, respectively, as compared to average of $C_{(x-x)}$ and $C_{(y-y)}$. Comparisons for heterogeneous pairs $\left(C_{(x-y)}\right)$ are made with average of $C_{(x-x)}$ and $C_{(y-y)}$ in Fig. 9. From this exercise, it can be concluded that LC between two different vehicle types cannot be considered as combined contribution of two similar types of vehicles' individual behaviors. There is a general shying away when interaction of dissimilar vehicle types is considered. The driver of one vehicle type is more confident of vehicle performance of all identical vehicle types in the stream and can estimate their movement with better accuracy than other vehicle types. Thus, he/she can take a higher risk and maintain lesser gap between his/her own vehicle and other vehicle of identical vehicle type.

\subsection{Effect of carriageway width on LC versus average speed relationship}

During manual vehicle identification of interacting vehicles through video camera, widths of carriageways were also denoted in terms of number of lanes of road $(N)$. 


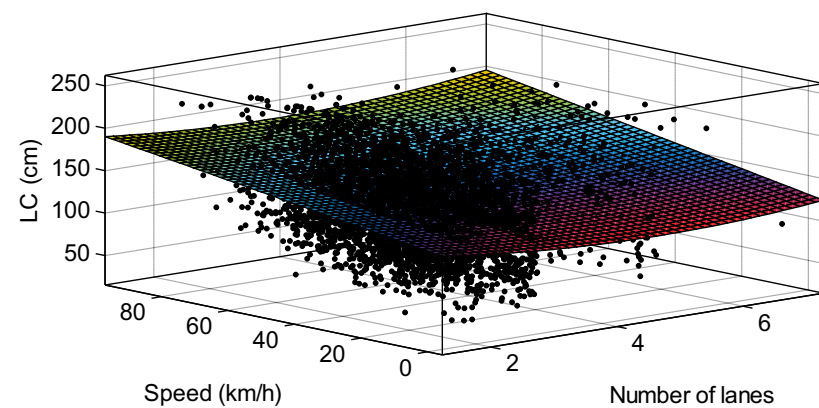

Fig. 10 Best-fitted regression plane of LC versus average speed relationship for pair of vehicles, varying with number of lanes of the road they move upon

Carriageway width varies from 1.5 lanes to 7 lanes. Vehicles are observed to utilize paved shoulders (of width $1.5 \mathrm{~m}$ ) as part of main carriageway. Therefore, paved shoulders are considered as half of lane width. Combined data for all vehicles are segregated widthwise, and a 3-d regression plane is fitted on $C, \bar{v}$, and road width data. Figure 10 provides the 3 -d regression plane, and Eq. (4) represents the relationship among average speed, LC and road width. It is observed that the LC decreases with decrease in carriageway widths (for similar speed levels) initially up to carriageways four lanes wide, and thereafter it increases. Second-degree equation with the number of lanes is chosen, since it provides a better fit as compared to linear equation. The aim of this analysis is to find the width where LC is minimum for a particular speed. Partial differentiation of best-fit regression surface with number of lanes $(N)$ (Eq. (4), second degree with $N$, first degree with $\bar{v})$ at zero speed obtains $N=4.59(\approx 4.5$ lanes $)$. At average speeds about $90 \mathrm{~km} / \mathrm{h}$, it obtains $N=4$ lanes. It means that on carriageways with 4-4.5 lane width, vehicles achieve maximum squeezing. The LC obtained can be calculated from Eq. (4).

$C=149-13.79 N+0.553 \bar{v}+1.5 N^{2}+0.02 \bar{v} N$.

Thus, a variation is observed between LC and width of road, primarily due to the effect of constraining by road edges or median. Due to this constraining, vehicles may not choose to overtake on narrow roads, and thus maintain larger LC at particular speeds. However, on very wide roads ( $>4$ lanes), lack of any constrain from road edges may motivate the drivers to travel at higher speeds taking higher risks, due to which LC again reduces at particular average speeds.

\subsection{Study of LC in case of multiple vehicle interactions}

Interaction of vehicles are decided based on vehicle's detection by the sensors fitted on both sides of the vehicles

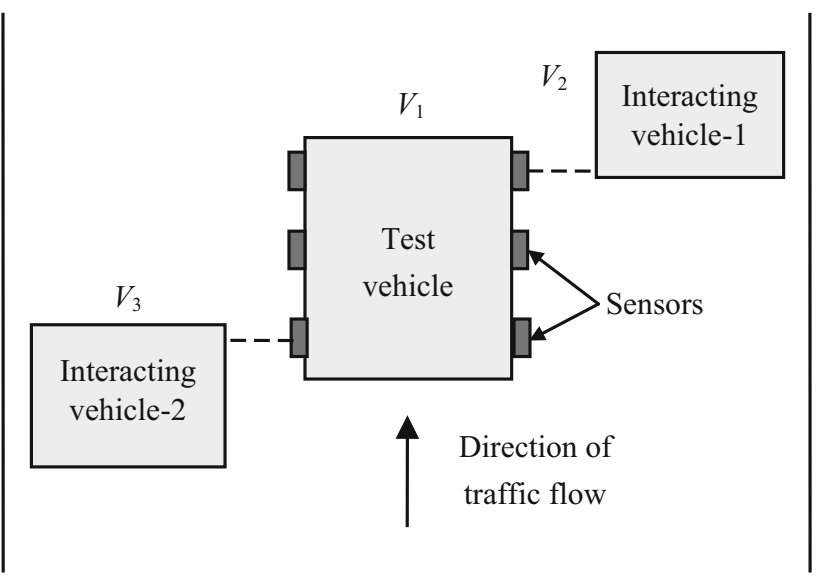

Fig. 11 Necessary condition of detecting interacting vehicles for multiple vehicle interactions

(refer to Fig. 2). The necessary condition for multiple vehicle interactions is that a vehicle should be detected by at least one sensor on one side of the test vehicle, while at least one sensor on the other side also detects another vehicle, at the same time step, as shown in Fig. 11.

There are four possible cases for lateral interactions observed when a vehicle traverses in a heterogeneous stream with weak lane discipline

Case 1: Interaction with vehicles is only on one side.

Case 2: Interaction is on both sides, and test vehicle is overtaking both the vehicles $\left(V_{1}>V_{2}, V_{1}>V_{3}\right.$ in Fig. 11).

Case 3: Interaction is on both sides, and test vehicle is getting overtaken by other two vehicles. $\left(V_{1}<V_{2}\right.$, $V_{1}<V_{3}$ in Fig. 11).

Case 4: Interaction is on both sides, and test vehicle is overtaking one vehicle and getting overtaken by other vehicle. $\left(V_{2}>V_{1}>V_{3}\right)$.

Case 1 is considered as unconstrained lateral interaction for analysis. Case 2 is considered as constrained lateral interaction. Cases 3 and 4 are ambiguous and not considered for comparison, since it is difficult to conclude whether the test vehicle is in constrained or unconstrained condition. The situation of test vehicle overtaking both vehicles by moving in the gap between them is a definite indicator of constrained lateral interaction. If Case 2 is observed, then interaction with both the vehicles is assigned as constrained interaction. Data of different vehicle pairs from Cases 1 and 2 are compared with each other. In order to avoid constraining due to median or road edges, the authors have removed data obtained from carriageways with a width of less than three lanes. Data for both the cases are modeled as per Eq. (2). Comparison of regression equations (slopes and intercepts) are made for some vehicle pairs (car-car, car-auto, car-bike and auto- 


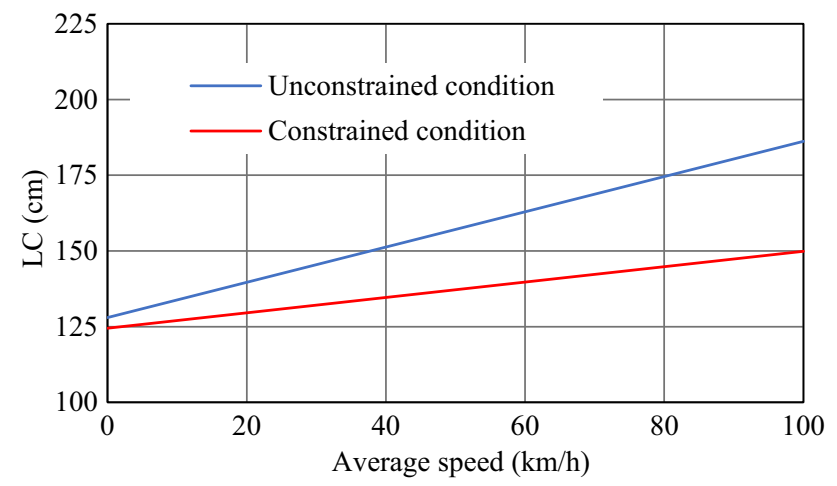

Fig. 12 Comparison of regression equations of LC-average speed relationship of car-car pair in constrained and unconstrained conditions

bike) with significant data for both the conditions. Both the regression equations are plotted in Fig. 12 for car-car interaction. The comparisons of slopes and intercepts of constrained conditions with unconstrained conditions for various pairs are presented in Table 5. The last column presents $p$-statistic of fit of beta distribution with actual field data.

From Table 5, it is observed that for all the vehicle pairs, there is a reduction in slopes of data for constrained conditions (by 18\%-80\%) when compared with unconstrained conditions. The highest reduction is observed for car-bike and auto-bike pairs. Slopes of data for constrained and unconstrained cases are compared with each other using ANCOVA test, and they are significantly different, except for car-car case at 5\% significance levels. ( $p=0.088,0.046,0.035$ and 0.041 for car-car, auto-car, car-bike and auto-bike, respectively). However, there is no significant difference in the intercepts between constrained and unconstrained relationships for any of the vehicle pairs. This may happen as at near zero speeds, LC requirement is quite low and vehicles really do not feel constrained at that speed with the existing lateral gap. From the residual

Table 5 LC versus average speed model for different pairs in constrained and unconstrained conditions

\begin{tabular}{|c|c|c|c|c|c|c|c|c|c|}
\hline \multirow[t]{2}{*}{ Vehicle pair } & \multirow[t]{2}{*}{ Condition } & \multirow[t]{2}{*}{ Sample size } & \multicolumn{2}{|c|}{ Regression line } & \multicolumn{5}{|c|}{ Parameters of residuals beta distribution } \\
\hline & & & Slope & Intercept & $\alpha_{1}$ & $\alpha_{2}$ & $a$ & $b$ & $p$-statistic \\
\hline \multirow[t]{2}{*}{ Car-Car } & Unconstrained & 1000 & 0.519 & 120.65 & 3.016 & 1.854 & -72.05 & 118.3 & 0.034 \\
\hline & Constrained & 197 & 0.381 & 125.19 & 2.154 & 1.379 & -67.96 & 106.96 & 0.054 \\
\hline \multirow[t]{2}{*}{ Auto-Car } & Unconstrained & 379 & 0.311 & 122.85 & 7.668 & 4.634 & -118.72 & 196.68 & 0.045 \\
\hline & Constrained & 112 & 0.256 & 135.26 & 2.712 & 2.085 & -96.63 & 125.80 & 0.059 \\
\hline \multirow[t]{2}{*}{ Car-Bike } & Unconstrained & 553 & 0.632 & 127.35 & 2.875 & 2.058 & -79.31 & 112.31 & 0.051 \\
\hline & Constrained & 141 & 0.26 & 135.68 & 2.193 & 1.339 & -82.83 & 58.63 & 0.055 \\
\hline \multirow[t]{2}{*}{ Auto-Bike } & Unconstrained & 151 & 0.864 & 126.46 & 1.276 & 1.322 & -87.57 & 86.59 & 0.075 \\
\hline & Constrained & 67 & 0.171 & 136.60 & 2.063 & 1.868 & -91.84 & 103.12 & 0.071 \\
\hline
\end{tabular}

Table 6 Evaluation of means of field and model data at different speed intervals for different vehicle pairs

\begin{tabular}{|c|c|c|c|c|c|c|c|}
\hline \multirow[t]{2}{*}{ Vehicle pair } & \multicolumn{7}{|c|}{ Speed range $(\mathrm{km} / \mathrm{h})$} \\
\hline & $0-10$ & $10-20$ & $20-30$ & $30-40$ & $40-50$ & $50-60$ & $60-70$ \\
\hline All combined & 0.294 & 0.338 & 0.187 & 0.052 & 0.045 & 0.362 & 0.874 \\
\hline Auto-Auto & N.D. & 0.762 & 0.886 & 0.178 & 0.355 & N.D. & N.D. \\
\hline Auto-Bike & 0.817 & 0.746 & 0.647 & 0.411 & 0.097 & N.D. & N.D. \\
\hline Auto-HV & 0.716 & 0.833 & 0.445 & 0.209 & 0.123 & 0.566 & N.D. \\
\hline Bike-Bike & N.D. & 0.710 & 0.667 & 0.314 & 0.789 & 0.077 & N.D. \\
\hline Car-Auto & 0.746 & 0.716 & 0.314 & 0.065 & 0.187 & 0.188 & N.D. \\
\hline Car-Bike & 0.098 & 0.184 & 0.174 & 0.188 & $\mathbf{0 . 0 3 1}$ & 0.657 & 0.435 \\
\hline Car-Car & 0.186 & 0.047 & 0.202 & 0.287 & 0.446 & 0.373 & 0.131 \\
\hline Car-HV & N.D. & 0.918 & 0.718 & 0.605 & 0.755 & 0.385 & N.D. \\
\hline Car-LCV & N.D. & N.D. & 0.863 & 0.164 & 0.373 & 0.547 & N.D. \\
\hline
\end{tabular}

N.D. indicates no data available for comparison

Bold values: significance level $p<5 \%$ 
(a) Car-Car (field data)

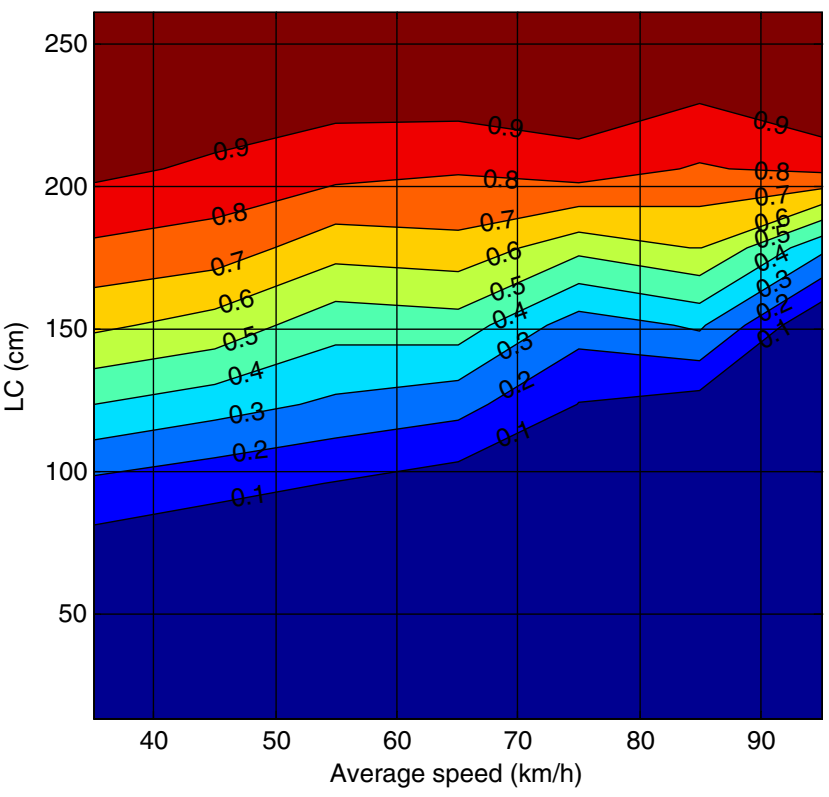

(c) Auto-Bike (field data)

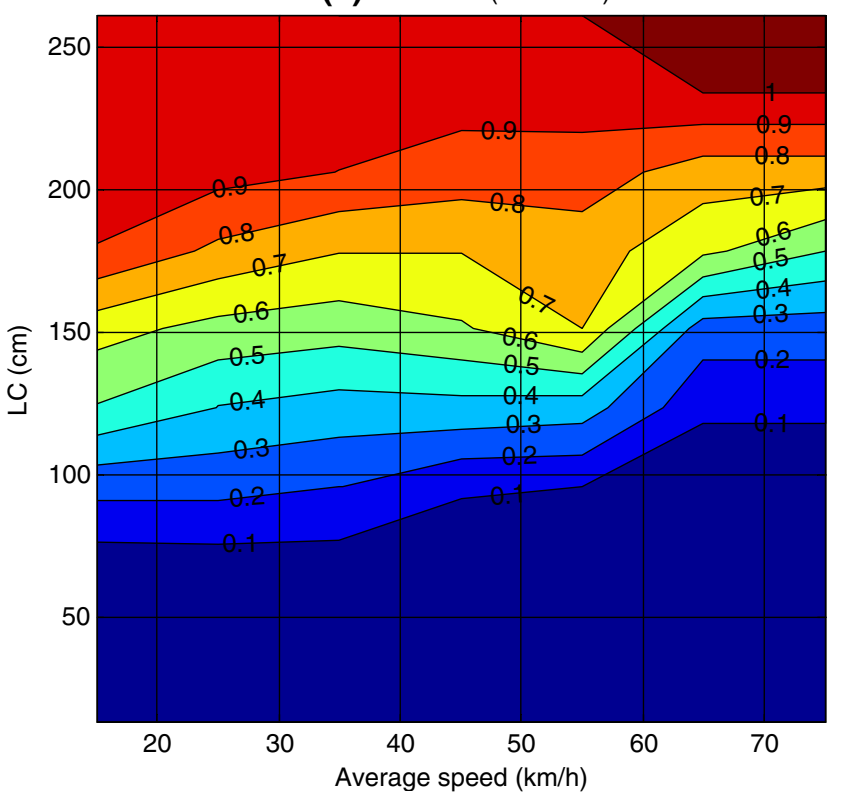

(b) Car-Car (model data)

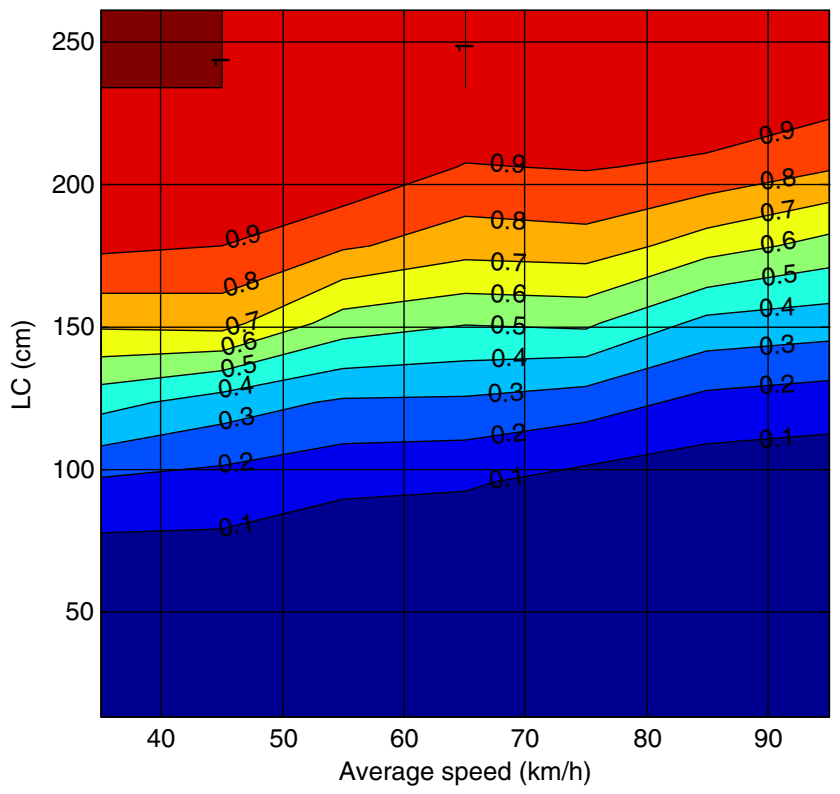

(d) Auto-Bike (model data)

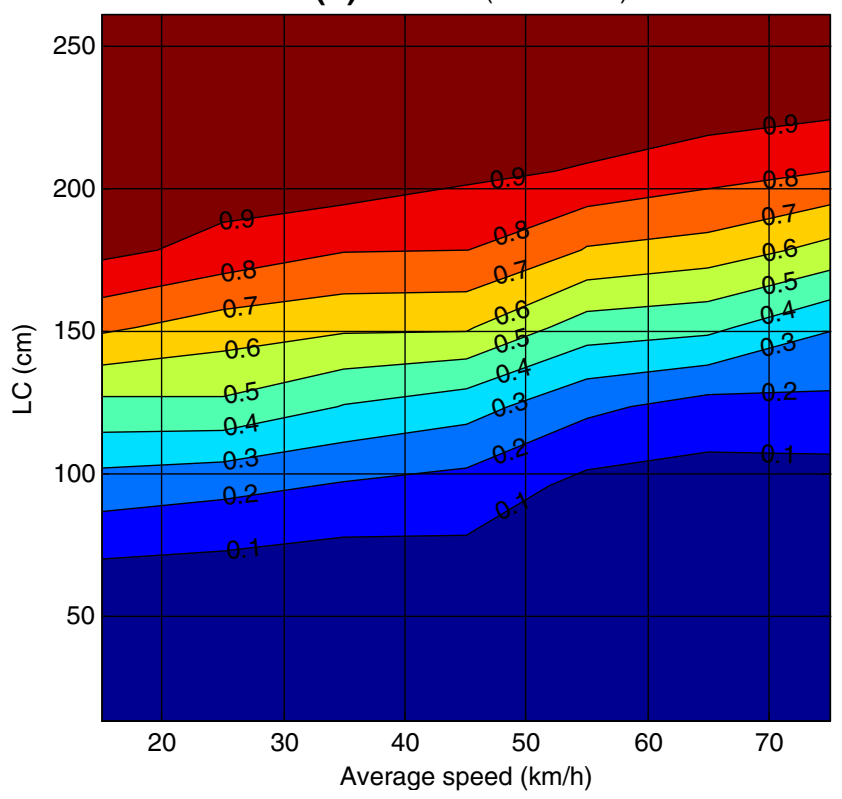

Fig. 13 Frequency plots for field data car-car (a), modeled data car-car (b), field data auto-bike (c), and modeled data auto-bike (d)

coefficients $a$ and $b$ of constrained and unconstrained cases, one can conclude that except for auto-bike pair, there is less spread of data about the regression line (indicated by difference between $a$ and $b$ ) for constrained case as compared with unconstrained case.

This comparative study reveals that vehicles compromise in LC (which also imply the safety of the vehicle) to a great extent at higher speeds during constrained overtaking.

\subsection{Evaluation of model quality and comparison with earlier literature}

The developed relationship between $C$ and $\bar{v}$ for different vehicle pairs can be used in car-following models or traffic simulators for better representation of vehicular interaction in heterogeneous traffic streams with weak lane discipline. Modeling of residuals in Eq. (2) can be incorporated by assigning a random risk factor to a particular vehicle 
Table 7 Comparison of LC values with previous researches for car-car pair

\begin{tabular}{lll}
\hline Research & \multicolumn{2}{l}{ Average LC (m) for car-car interaction } \\
\cline { 2 - 3 } & $0 \mathrm{~km} / \mathrm{h}$ & $50 \mathrm{~km} / \mathrm{h}$ \\
\hline This paper & 1.173 & 1.581 \\
Arasan and Koshy [12] & 0.600 & 1.000 \\
Mallikarjuna et al. [3] & $0.85^{\mathrm{a}}$ & $1.500^{\mathrm{a}, \mathrm{b}}$ \\
Dimayacyac and Palmiano [21] & 0.500 & 1.470 \\
Gunay [8] & $0.800^{\mathrm{a}}$ & $1.600^{\mathrm{a}}$ \\
\hline
\end{tabular}

${ }^{a}$ Estimated value

b Extrapolated value

(irrespective of its vehicle type), which is beta-distributed. This risk factor can be an indicator of driver's aggressiveness based upon his/her driving experience, physical and mental state and other factors. Beta-distributed random numbers can be generated equal to a desired number of vehicles, as residuals into Eq. (2). Values of intercept, slope of $C$ versus $\bar{v}$ regression line and the parameters of beta distribution for particular vehicle pair are substituted for a particular $\bar{v}$, using Table 4 .

The model quality needs to be evaluated to check how close the developed model is to the original field data. For this purpose, vehicle pairs are generated as per the description in the above paragraph, and the means of field data and generated modeled data are compared at various speed intervals using $T$ test. The comparison is presented in Table 6. For evaluating spread of data across the mean, $p$ statistic was evaluated between residual plots of model and field, and already mentioned in last column of Table 4. It can be concluded that null hypothesis (that there is significant similarity between model and field data) fails to be rejected at all speed intervals. There are lower $p$ values for 'All combined' data since all combined data represent a mix of vehicle pairs.

For sample representation of difference between modeled and field relationships, data points from model of carcar and auto-bike relationships are generated. Further, a frequency matrix based on the number of data points present corresponding to each $10 \mathrm{~km} / \mathrm{h}$ speed interval and $25 \mathrm{~cm}$ LC interval groups is generated for both modeled and field data. Contour plots of LC versus average speed for both vehicle pairs (car-car and auto-bike) are generated based on the corresponding frequency matrix and presented in Fig. 13a-d. Numbers on the contour lines in the chart represent the fraction of vehicles maintaining LC lower than the particular contour line. Fraction values (i.e., relative frequencies of LC at a particular speed range) are used since data for lower and higher speed ranges are different. These charts compare graphically the modeled and field data of LC for pairs car-car and auto-bike. For example, for auto-bike field data (Fig. 13c), at speed $40 \mathrm{~km} / \mathrm{h}$, about 0.6 fraction of total vehicles maintain LC less than $150 \mathrm{~cm}$. The modeled data from Fig. 13d also reflect similar behavior. LC comparison of other vehicle pairs can also be made in a similar manner. The model is able to reflect the spread and variation in $\mathrm{LC}$ with average speed.

Previous researchers have calculated inter-vehicular gaps using static data-collection techniques (such as video recording), whereas this paper uses moving observer method to calculate these gaps. Average value over the entire overtaking duration is not captured due to limitations on trap length in video-recording techniques. Table 7 presents the comparison of average LC values for car-car pair as obtained from this paper with previous researches. The LC matches with the data from Mallikarjuna et al. [3] as well as Gunay [8], due to similarity in traffic behavior of studied traffic in these researches to that studied in this paper. The values estimated by Dimayacyac and Palmiano [21] are showing higher sensitivity to speed, since the traffic studied was more organized and followed lane discipline.

\section{Conclusion}

Lateral interactions between vehicles in heterogeneous traffic with weak lane discipline (generally observed in developing countries) are studied in this paper. Instrumented test vehicles fitted with GPS, camera and sensors are developed, and LCs maintained by interacting vehicles with these test vehicles are measured on roads located in six metro cities of India. Obtained LCs with different interacting vehicle pairs are modeled as a deterministic part (regression line) and a stochastic part (residual distribution). These relationships are studied with changing various vehicle pairs, road widths and introduction of constraining in overtaking, and compared with each other. The following conclusions can be derived from this study: 
- LC $(C)$ versus average speed $(\bar{v})$ relationship of interacting vehicle pairs follows an upward linear trend with Beta-distributed residual; i.e., LC increase with an increase in interacting vehicles speed. Similar trend is observed in data collected from all six cities.

- $C$ versus $\bar{v}$ relationships are modeled for various vehicle pairs such as car-car, car-bike and bike-auto. From the information of regression lines for models of various pairs, it is observed that motorized two-wheelers (bikes) maintain the least LC. Cars maintain the highest LC with light commercial vehicles (LCVs). Vehicles maintain lesser LC with heavy vehicles than with their own vehicle types due to their poor acceleration characteristics and maneuverability. For similar reasons, vehicles maintain higher clearances with autos.

- LC maintained with the same vehicle type is lesser than that maintained with different vehicle types at similar speed levels. Thus, the LC between a pair of different vehicle types cannot be considered as the average of LC between the pairs of corresponding similar vehicle types.

- It is observed that vehicles achieve maximum squeezing-in at a carriageway width of four lanes with paved shoulders.

- When a vehicle interacts with multiple vehicles simultaneously, there is a compromise on LC at a particular average speed. The slope of LC versus average speed changes from $18 \%$ to $85 \%$ for different vehicle types in constrained versus unconstrained condition. However, intercepts remain consistent, indicating compromise in safety at higher speed ranges only.

The lateral interactions studied in this paper can be combined with longitudinal or car-following behavior with lateral discomfort, in order to model weak lane discipline traffic. A model with car-following, overtaking decisionmaking and LC values (from this paper) together will help in accurate estimation of behavior and simulation of heterogeneous traffic with weak lane discipline.

Open Access This article is distributed under the terms of the Creative Commons Attribution 4.0 International License (http:// creativecommons.org/licenses/by/4.0/), which permits unrestricted use, distribution, and reproduction in any medium, provided you give appropriate credit to the original author(s) and the source, provide a link to the Creative Commons license, and indicate if changes were made.

\section{References}

1. Gunay B (2007) Car following theory with lateral discomfort. Transp Res Part B 41(7):722-735

2. Pal D, Mallikarjuna C (2010) Cellular automata cell structure for modeling heterogenous traffic. Transporti Europei 45:5063
3. Mallikarjuna C, Tharun B, Pal D (2013) Analysis of the lateral gap maintaining behavior of vehicles in heterogeneous traffic stream. In: 2nd CTRG, Agra, India, 2013

4. May A (1959) A friction concept of traffic flow. In: Proceedings, 30th annual meeting of the highway research board, National Academy of Science-NRC, Washington, pp 493-510

5. Mahalel D, Hakkert A (1980) Traffic arrival patterns on a cross section of a multilane highway. Transp Res Part A 17(4):263-270

6. Nagraj B, George K, John P (1992) A study of linear lateral placement of vehicles in mixed traffic environment through video-recording. Highw Res Bull Indian Road Congr 42:105-136

7. Gunay B (2003) Methods to quantify the discipline of lane-baseddriving. J Traffic Eng Control 44(1):22-27

8. Gunay B (2009) Rationality of a non-lane-based car-following theory. Proc ICE Transp 162(1):27-37

9. Gunay B, Erdemir E (2011) Lateral Analysis of longitudinal headways in traffic flow. IJEAS 3(2):90-100

10. Nair R, Mahmassani H, Miller-Hooks E (2011) A porous flow approach to modeling heterogeneous traffic in disordered systems. Transp Res Part B 45(9):1331-1345

11. Ambarwati L, Pel A, Verhaeghe R, Arem B (2014) Empirical analysis of heterogeneous traffic flow and calibration of porous flow model. Transp Res Part C 48:418-436

12. Arasan V, Koshy R (2005) Methodology for modeling highly heterogeneous traffic flow. J Transp Eng 131(7):544-551

13. Maurya A, Chakroborty P (2007) CASIM: A realistic CA based traffic flow model. In: Proceedings of 11th World conference on transportation research, Berkeley, California, USA

14. Metkari M, Budhkar A, Maurya A (2013) Development of simulation model for heterogeneous traffic with no lane discipline. In: Proceedings of the 2nd CTRG, Agra, India, pp 360-369

15. Chakroborty P, Agrawal S, Vasishtha K (2004) Microscopic modeling of driver behavior in uninterrupted traffic flow. ASCE J Transp Eng 130(4):438-451

16. Delpiano R, Herrera M JC, Coeymans A JE (2015) Characteristics of lateral vehicle interaction. Transp A 11(7):636-647. doi:10.1080/23249935.2015.1059377

17. Jin S, Wang D, Tao P, Li P (2010) Non-lane-based full velocity difference car following model. Phys A 389(21):4654-4662

18. Jin S, Wang D, Yang X (2011) Non-lane-based Car-following Model with Visual Angle In formation. Transp Res Rec J Transp Res Board 2249(1):7-14

19. Pal D, Mallikarjuna C (2016) Analysis of the effect of variable lateral gap maintaining behavior of vehicles on traffic flow modeling. Procedia Eng 142:198-204

20. Munigety C, Mantri S, Mathew T, Rao K (2014) Effect of surrounding traffic characteristics on lateral movement behaviour in heterogeneous traffic conditions. In: Proceeding of 92nd annual transportation research board (TRB) meeting, Washington, USA

21. Dimayacyac E, Palmiano H (2016) Calibrating relative velocity and lateral clearance parameters of a lane changing model for traffic microsimulation. In: Proceedings of 23rd annual conference of the Transportation Science Society of the Philippines Quezon City, Philippines, 8 Aug 2016

22. PVT Group (2016) PVT Vissim. http://vision-traffic.ptvgroup. com/en-us/products/ptv-vissim/

23. Wong C, Qidwai U (2004) Vehicle collision avoidance system [VCAS]. Sensors. In Proceedings of IEEE, pp 316-319

24. Venter C, Knoetze H (2013) Lateral clearance between vehicles and bicycles on urban roads. In: 32nd South African transport conference (SATC 2013)

25. Sun Z, Bebis R, Miller G (2004) On-road vehicle detection using optical sensors: a review. In: Proceedings 7th international IEEE conference on intelligent transportation systems, Washington, USA 